\title{
Fracture-Based Fabrication of Normally Closed, Adjustable, and Fully Reversible Microscale Fluidic Channels
}

\author{
Byoung Choul Kim, Christopher Moraes, Jiexi Huang, Toshiki Matsuoka, \\ M. D. Thouless, * and Shuichi Takayama*
}

\begin{abstract}
Adjustable fluidic structures play an important role in microfluidic systems. Fracture of multilayered materials under applied tension has been previously demonstrated as a convenient, simple, and inexpensive approach to fabricate nanoscale adjustable structures; here, it is demonstrated how to extend this concept to the microscale. This is achieved by a novel pairing of materials that leverages fracture mechanics to limit crack formation to a specified region, allowing to create size-controllable and adjustable microfluidic structures. This technique can be used to fabricate "normally closed" microfluidic channels that are completely reversible, a feature that is challenging to achieve in conventional systems without careful engineering controls. The adjustable microfluidic channels are then applied to mechanically lyse single cells, and subsequently manipulate the released nuclear chromatin, creating new possibilities for epigenetic analysis of single cells. This simple, versatile, and robust technology provides an easily accessible pathway to construct adjustable microfluidic structures, which will be useful in developing complex assays and experiments even in resource-limited settings.
\end{abstract}

\section{Introduction}

Adjustable fluidic structures are of critical importance in the control and manipulation of fluids at the microscale. The use of these structures as valves, ${ }^{[1,2]}$ flow controllers, ${ }^{[3]}$ and pumps $^{[4]}$ has significantly improved microfluidic throughput, ${ }^{[5]}$ automation, ${ }^{[6]}$ and sorting and trapping capabilities, ${ }^{[7]}$ particularly for biological applications. However, fabricating adjustable structures is typically a challenging process, requiring unconventional fabrication techniques or

\author{
B. C. Kim, C. Moraes, T. Matsuoka, S. Takayama \\ Department of Biomedical Engineering \\ College of Engineering \\ University of Michigan \\ 2200 Bonisteel Blvd, Ann Arbor, MI 48109, USA \\ E-mail: takayama@umich.edu \\ B. C. Kim, S. Takayama \\ Macromolecular Science and Engineering Center \\ College of Engineering \\ University of Michigan \\ 2300 Hayward St., Ann Arbor, MI 48109, USA
}

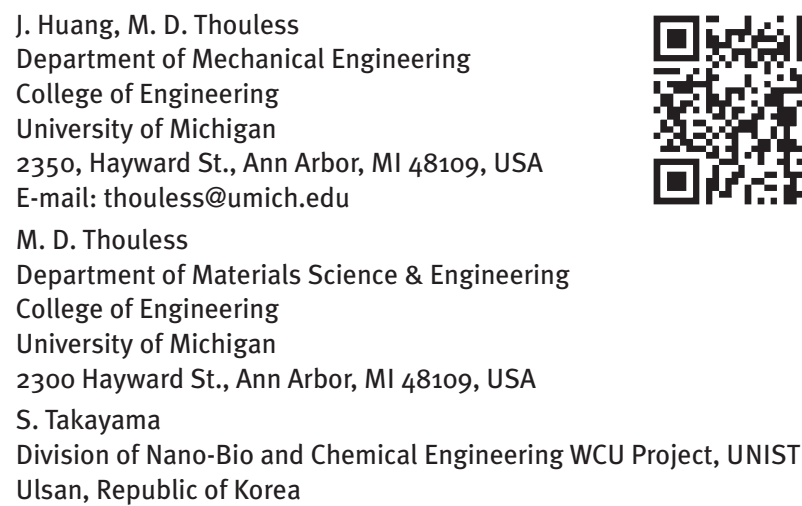


precisely controlled actuation mechanisms. Given that biological applications generally require robust, reliable and precise control of microstructures, there exists a need for simple technologies that enable dynamic manipulation of micrometer-scaled features.

Previous work in our lab has focused on the fabrication of adjustable nanoscale fluidic channels by the formation of stable arrays of cracks in multilayered materials under applied tensile strains. ${ }^{[8-13]}$ In these studies, a thin brittle layer of oxidized PDMS is sandwiched between two tough PDMS slabs, and fractured to form stable nanofluidic crack structures. This nanofabrication approach is simple, robust and versatile; we and others have used these technologies for various biomedical applications. ${ }^{[14,15]}$ These have included the use of fractured nanofluidic channels to mechanically elongate DNA for epigenetic analysis of chromatin, ${ }^{[11]}$ and to sort and trap nanoparticles. ${ }^{[9]}$ An open crack configuration has also been used as adhesive protein matrices for cell culture. ${ }^{[8,12,16]}$ While the utility of this nano-crack technology has been established for handling and patterning nanoscaled structures, the ability to extend this technology broadly into the micrometer-scale regime could be particularly fruitful. For example, such techniques would enable the transport of sufficient quantities of reagents to maintain and stimulate cultured cells: a prerequisite for most microfluidic cells-onchip applications. In this work, we build on our previous experience with fracture-based fabrication techniques to develop a reliable and versatile system that generates predictable, adjustable and fully reversible fluidic features at the scale of micrometer.

The characteristic dimensions of fracture-fabricated structures in oxidized PDMS/PDMS systems are typically limited to $<1 \mu \mathrm{m} .{ }^{[17]}$ This limitation arises because cracks formed in this system do not significantly tunnel into the underlying PDMS, and the crack depth is limited to the thickness of the oxidized PDMS. Fabricating oxidized layers thicker than $1 \mu \mathrm{m}$ is challenging as the layer has a mismatched thermal coefficient with the substrate, and sample heating associated with the plasma oxidation process causes the spontaneous formation of undesired cracks. ${ }^{[18,19]}$ Hence, it is challenging to use this system to extend the crack features beyond submicrometer dimensions.

An alternative approach, recently explored by our group $^{[20-22]}$ and others, ${ }^{[6,23,24]}$ is the use of a deposited metal layer of gold on bulk PDMS. The huge modulus mismatch with the PDMS substrate prompts the formation of cracks that tunnel into the PDMS. The depth to which these cracks grow is dependent on the distance between neighboring cracks, as well as on the applied strain and modulus/toughness mismatch between the materials. ${ }^{[25]}$ Although this system may be used to generate micrometer-scaled crack structures, the crack dimensions cannot be reliably controlled, and exhibit broad variability. Moreover, this approach requires physical vapor deposition systems available only in specialized cleanrooms, and the devices are not optically transparent, preventing use of these systems with conventional inverted microscopes.

In order to provide a versatile, tunable system in which crack fabrication can be used to generate a broad variety of structures at the micrometer scale, we present a novel material pairing that allows us to define the crack depth and width independently at this scale. Our strategy is based on preventing cracks from propagating into the substrate by selecting a material for the brittle layer with appropriate mechanical properties that can be deposited as a film of micrometer-scale thickness. Hard PDMS (h-PDMS) ${ }^{[26]}$ is a variant of PDMS that can be spin-coated onto the PDMS substrate, and is brittle enough to initiate cracking. However, in contrast to gold, h-PDMS has a relatively small modulus mismatch with the substrate and, therefore, strongly localizes cracks to the surface layer. Hence, h-PDMS can be used to generate crack structures with well-defined dimensions at the micrometer-scale. We established that these structures are robust, adjustable, and completely reversible. We demonstrated the utility of this approach in designing "normally closed" adjustable microfluidic channels. We then used this technology to mechanically lyse and release chromatin from single cells for epigenetic analysis. This was achieved because of the unique capabilities of the system to allow a channel at the micrometer-scale to collapse all the way to a completelyclosed configuration. By opening the channels to the micrometer scale, individual cells can be positioned within them. These cells can then be mechanically lysed by collapsing the micro-channels to release nuclear chromatin. Further collapse of the channels through the nanoscale induces elongational shear flow that linearizes the chromatin complex. ${ }^{[11]}$

\section{Results and Discussion}

\subsection{Mechanical Characterization of Materials}

h-PDMS is known to be stiffer and more brittle than conventional PDMS. ${ }^{[26,34]}$ Tensile and compressive characterization tests confirmed these findings; the moduli were $3.7 \pm 0.3 \mathrm{MPa}$ for the PDMS and $9.2 \pm 0.6 \mathrm{MPa}$ for the h-PDMS. The mode-I toughness for the same PDMS used in this study was reported by Mills et al. ${ }^{[17]}$ to be $460 \pm 50 \mathrm{~J} / \mathrm{m}^{2}$. The toughness of the h-PDMS was determined to be $12.9 \pm 2.7 \mathrm{~J} / \mathrm{m}^{2}$ (all mechanical characterization data are summarized in Table 1). These data of a comparable modulus and a reduced toughness for the h-PDMS compared to the PDMS are important because they mean that cracks in the multi-layer system will essentially be localized to the h-PDMS film layer, rather than propagating significantly into the underlying PDMS substrate. ${ }^{[25,35]}$ Furthermore, the toughness of the h-PDMS is significantly higher than the toughness of the oxidized PDMS $\left(0.1-0.3 \mathrm{~J} / \mathrm{m}^{2} ;{ }^{[17]}\right.$ this avoids the problems of spontaneous cracking from thermal mismatch that occurs with thicker oxidized films.

Table 1. Mechanical characterization of materials

\begin{tabular}{lcc}
\hline & PDMS & h-PDMS \\
\hline Young's modulus $[\mathrm{MPa}]$ & $3.7 \pm 0.3$ & $9.2 \pm 0.6$ \\
Mode I toughness $\left[\mathrm{J} / \mathrm{m}^{2}\right]$ & $460 \pm 50^{\mathrm{a}}$ & $12.9 \pm 2.7$ \\
\hline
\end{tabular}

a) Data previously published by Mills et al. ${ }^{[17]}$ 


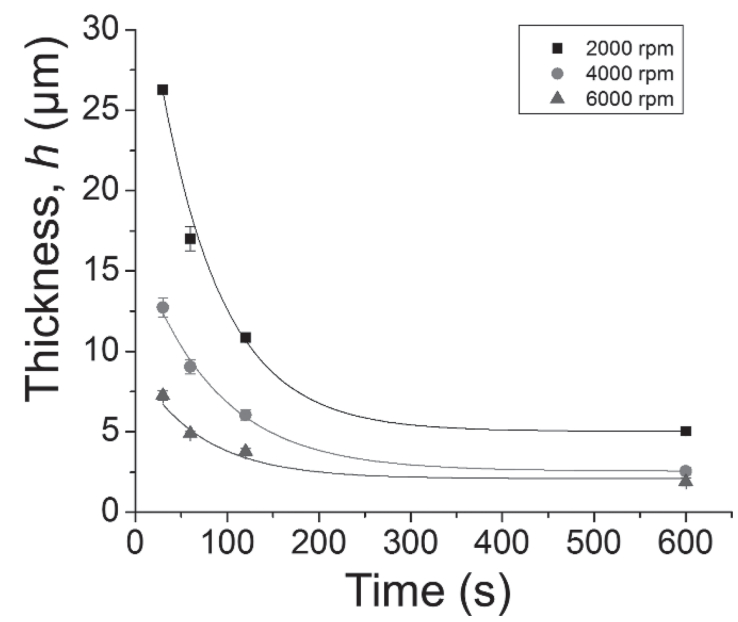

Figure 1. Characterization of spin coating parameters on h-PDMS thickness $(h)$. Spin speeds were varied from 2000 to 4000 RPM for between $30 \mathrm{~s}$ and $10 \mathrm{~min}$. This domain of parameters produces films that range from $<1 \mu \mathrm{m}$ to $27 \mu \mathrm{m}$ in thickness.

\section{2. h-PDMS Processing and Biocompatibility}

The ability to fabricate a thin film of a precisely defined thickness is an important feature in PDMS processing ${ }^{[6,28]}$ and, in this particular application, the thickness of the h-PDMS is a critical parameter as it dictates the crack depth. Hence, the relationship between spin parameters and film thickness was carefully characterized for h-PDMS (Figure 1). As expected, spin speed plays a significant role in defining the film thickness, and the film thickness exponentially decreases towards a lower limit with spin time. The use of hexane as a solvent to reduce the viscosity and, hence, the film thickness of h-PDMS was also characterized (Supporting Information Figure S1); but this was found not to make a substantial difference over the range of hexane/PDMS dilutions tested, particularly for longer spin times. This is likely caused by increased solvent evaporation during extended spin times. These results confirm that thin films of h-PDMS can be processed in a manner compatible with PDMS fabrication processes, and a range of film thicknesses from less than $1 \mu \mathrm{m}$ to greater than $20 \mu \mathrm{m}$ can be produced. For all further studies discussed in this paper, a spin speed of 6000 RPM was used, and the spin times were varied to control the h-PDMS film thickness.

To ensure that h-PDMS retained the advantages of PDMS for any potential biological applications, we conducted a simple biocompatibility study to test cell adhesion to h-PDMS surfaces. Fibroblasts adopted well-spread morphologies after one day in culture (Supporting Information Figure S2), and showed no unusual phenotypes. This data strongly suggests that h-PDMS may be used without unusual surface modifications for biological cell-culture applications.

\subsection{Characteristics of Crack Profiles}

Cracks were formed in multilayered materials by application of mechanical force. Strains were applied to devices using a commercially available MicroVice stage. The use of a simple tensile stage to actuate adjustable microfluidic structures provides some significant advantages over other actuation technologies, such as pneumatic- or hydraulically operated devices. The stage is stable, robust, inexpensive, easily transported, independent of external power supplies, and bypasses the need for extremely robust world-to-chip connections, a common source of failure in conventionally operated microfluidic devices. Strains up to $60 \%$ could be applied to the PDMS samples without them breaking.

The average spacing between cracks, $S$, in systems without V-notches, but with different film thicknesses, $h$, were measured (under strain) to confirm that the cracks were limited to the h-PDMS film. ${ }^{[25,27]}$ Although all data presented in this work were for cracks of length $5 \mathrm{~mm}$, as dictated by the relevant width of the samples, we have demonstrated that crack lengths in excess of $10 \mathrm{~mm}$ could be generated (Supporting Information Figure S3). The length of the cracks appears to be limited only by the ability of the rig to apply uniform strains across the sample surface. As shown in Supporting Information Figure S4, the non-dimensional straincorrected average crack spacing, $S / h\left(1+\varepsilon_{\mathrm{o}}\right)$, scales with the non-dimensional parameter $\varepsilon o\left(E_{\mathrm{f}} h / \Gamma_{\mathrm{f}}\right)^{1 / 2}$ (where $\varepsilon_{0}$ is the applied strain, and $E_{\mathrm{f}}$ and $\Gamma_{\mathrm{f}}$ are the modulus and toughness of the h-PDMS). This is the result expected from the fracturemechanics of thin films when the crack depth is limited to the film thickness, and there is no delamination at the interface.

To quantify the degree to which cracks are localized to the h-PDMS, increasing levels of strain were applied to systems with different thicknesses of h-PDMS films, and the crack depth (distance from the center of the crack trough to the imaginary line connecting the crack tips) was quantified using laser surface profilometry (Figure 2A). The crack depth was found to be relatively stable up to $60 \%$ applied strain, at which point the supporting PDMS layer underwent catastrophic failure. Small changes in the crack depth and variations in crack profile at higher strains suggest that cracks do penetrate slightly into the underlying PDMS layer, but the degree to which this occurs is relatively small, consistent with the slightly larger modulus of the cracked layer. ${ }^{[25]}$ Surface profilometry also revealed that the cracks had relatively flat profiles (Figure 2B, C; blue data points).

To confirm the mechanics underlying these dual observations of slight penetration and flat-bottomed crack profiles, numerical finite-element calculations were conducted. The relatively flat bottom of the crack profile suggests that the strain at the tip of the cracks was huge. Therefore the full stress-strain behavior of PDMS allowing for large deformations of the material was used in the simulations. ${ }^{[17]}$ The simulated penetration depth, $a / h$, was iteratively varied to give the best match to the experimentally measured crack profiles. Comparisons between the simulation results and experimental measurements (Figure 2B,C) indicate that $a / h=1.10$ at $25 \%$ applied strain and $a / h=1.14$ at $35 \%$ applied strain. The small increase in penetration with a large increase in strain is consistent with experimental observations, and quantifies the close relationship between the thickness of the h-PDMS film and the depth of the generated crack. (It should be emphasized that even if a film is only slightly stiffer than a substrate, cracks are expected to extend slightly across the interface and into the substrate. However, consistent with the experimental 

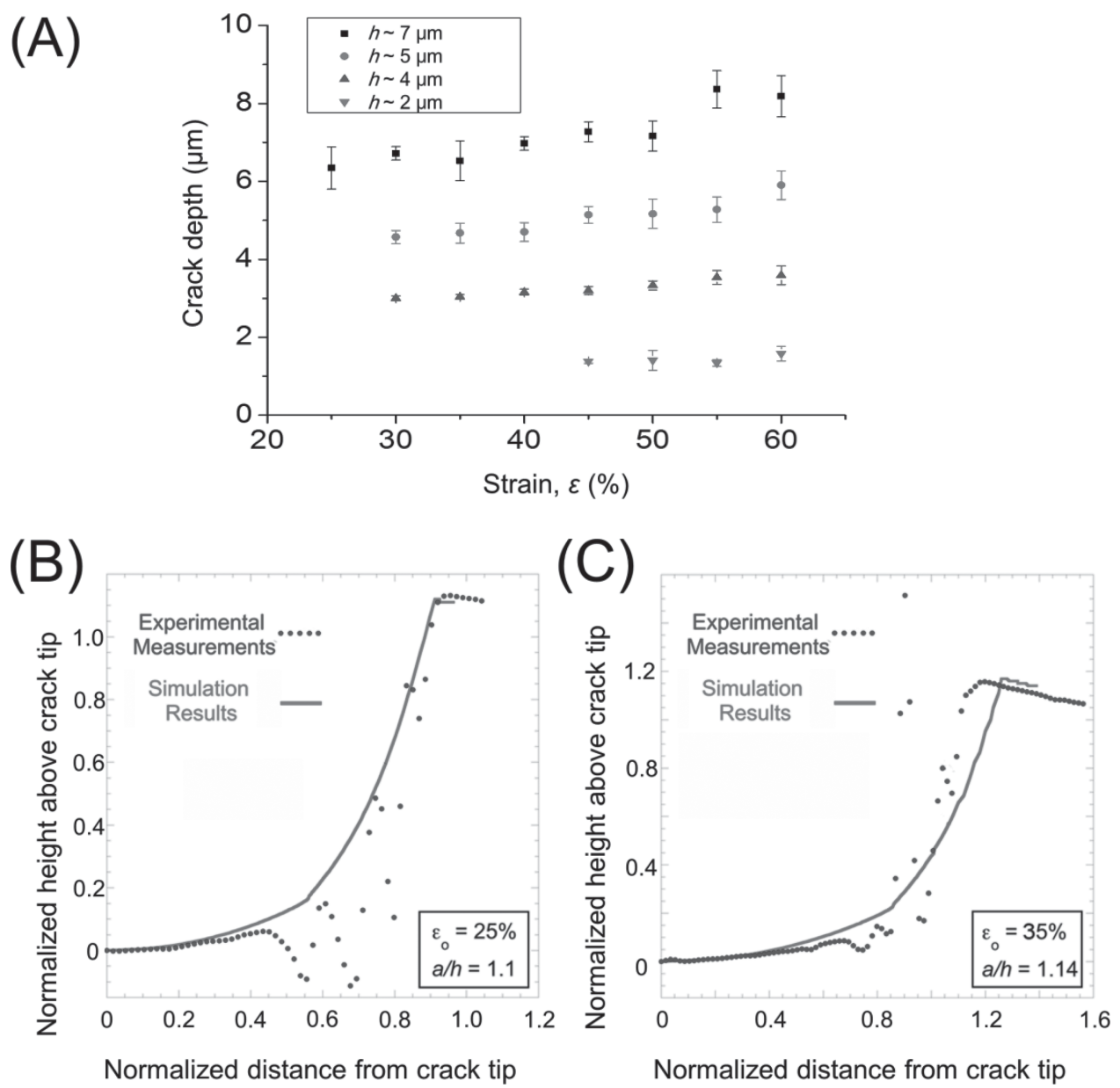

Figure 2. Characterization of crack depths in the h-PDMS/PDMS material system for various thicknesses of the brittle h-PDMS layer. A) The characteristic depths of the cracks remain stable and well-controlled over a broad range of applied strains; they closely match the thickness of the spin-coated h-PDMS layer. Small increases in crack depth with increasing applied strain suggests that some limited propagation of the crack into the underlying PDMS layer does occur. B,C) Finite-element simulations demonstrate that a hyperelastic model of the PDMS/h-PDMS system under applied tension matches crack profiles measured by laser scanning profilometry. The $x$ - and $y$-axes of the graph indicate the lateral distance from the crack tip, and the vertical profile of the crack respectively, both normalized to the thickness of the h-PDMS layer. A limited degree of penetration of the crack into the PDMS layer is expected, and was iteratively determined to match experimental data. The ratio between penetration depth and crack thickness $(a / h)$ was found to be B) 1.10 for an applied strain of $25 \%$, and C) 1.14 for an applied strain of $35 \%$. The hyperelastic material model predicts dramatic deformation in the PDMS at the crack tip to generate the relatively flat bottom of the crack profile. The noise in the experimental data was generated by the interaction between the laser-scanning profilometer and the crack side walls of the crack; it was ignored for fitting purposes.

observations, this penetration is expected to be insignificant for small modulus mismatches and tough substrates. $)^{[25]}$

To determine the reversibility of the system, the crack profiles were monitored using laser surface profilometry during cycles of applied load (Figure 3). These measurements revealed that the cracks appeared to have healed perfectly when allowed to close, with healing presumably associated with a physical attraction such as the van der Waals force. The complete reversibility of the system confirms that there was no delamination at the interface. This was further confirmed by experiments described in section 3.5, in which cracks formed in h-PDMS sandwiched between two PDMS layers were loaded with dye, and no apparent penetration into the interface is observed. Furthermore, cyclic strains were applied and released repeatedly; this resulted in fully reversible and completely-closed cracks, without additional damage to the material.
Taken together, these results demonstrate that the depths of generated cracks can be fairly accurately controlled by specifying the thickness of the h-PDMS layer. Once a crack has been formed, its width can be controlled by varying the applied strain, both to open and close it. The fact that the crack dimensions can be varied in such a fashion suggests that this material system is suitable for the generation of well-controlled and adjustable microscale fluidic channels. In principle, even larger channels can be generated by thicker layers of hPDMS.

\subsection{Predictive Control of Crack Position and width}

As is the case with conventional oxidized-PDMS/PDMS multilayer systems, it is challenging in the present h-PDMS/PDMS system to control precisely the location of individual cracks 


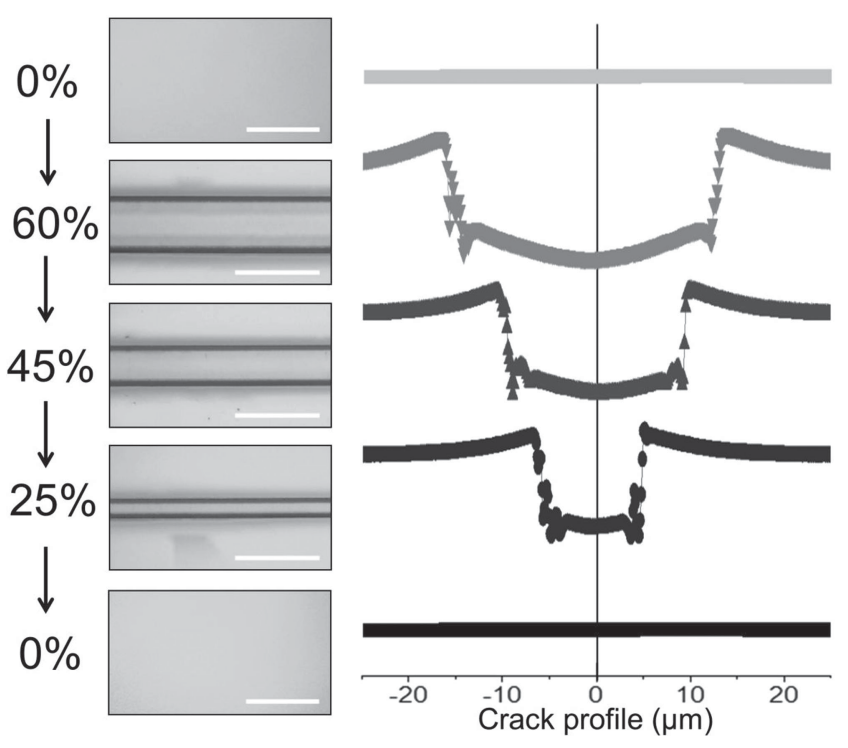

Figure 3. Cracks formed in h-PDMS/PDMS substrates reversibly close when the applied tension is removed. Optical images and highresolution laser-based surface profilometry of single cracks are unable to detect surface features after a crack is formed and closed, indicating that cracks are completely reversible, and that no delamination occurs between the layers. Scale bar $=50 \mu \mathrm{m}$.

because of the statistical nature of the intrinsic flaws responsible for initiating channeling cracks (see the error bars in Supporting Information Figure S3). Since the width of a crack is sensitive to the distance to its nearest neighbors, ${ }^{[25]}$ it is important to control the crack spacing if one wishes to control the crack width in adjustable microfluidic systems. Furthermore, control of crack location will be necessary in utilizing this approach for specific microfluidic applications. Therefore, we adapted an approach we developed recently to selectively activate intrinsic flaws in the h-PDMS material using microfabricated crack-initiating structures. ${ }^{[21]}$ To demonstrate this capability in the present system, we micro-fabricated V-notches spaced $700 \mu \mathrm{m}$ apart and incorporated them into the h-PDMS layer. These notches shield intrinsic flaws from the applied stress field, leaving only the flaws at their tips to be active and available to initiate a crack at the desired level of strain. ${ }^{[35]}$ As a result, cracks channel across the film from the tips of these notches (Supporting Information Movie 2). For h-PDMS layers of 5-11 $\mu \mathrm{m}$ in thickness, cracks only formed at these pre-specified sites for applied strains ranging from 5 to $25 \%$ (Figure 4A). In order to prevent additional randomly positioned cracks, the design of the $\mathrm{V}$-notch spacing can be adjusted according to the desired strains and thickness of the h-PDMS, as previously described. ${ }^{[21,35]}$ This simple demonstration indicates that the position of crack-generated microchannels can be specified a priori as part of the design process.

As expected, using the V-notch system to control spacing between cracks, allows the crack profiles to be controlled fairly accurately. The crack width can be controlled up to $50 \mu \mathrm{m}$, and depends upon the thickness of the h-PDMS film and the applied strain (Figure 4B). Some variation in widths on the spacing-controlled systems were observed with the h-PDMS/PDMS system possibly due to small localized variations in mechanical properties of the h-PDMS polymer layer. The widths of the cracks generated in this system are of the same order of magnitude
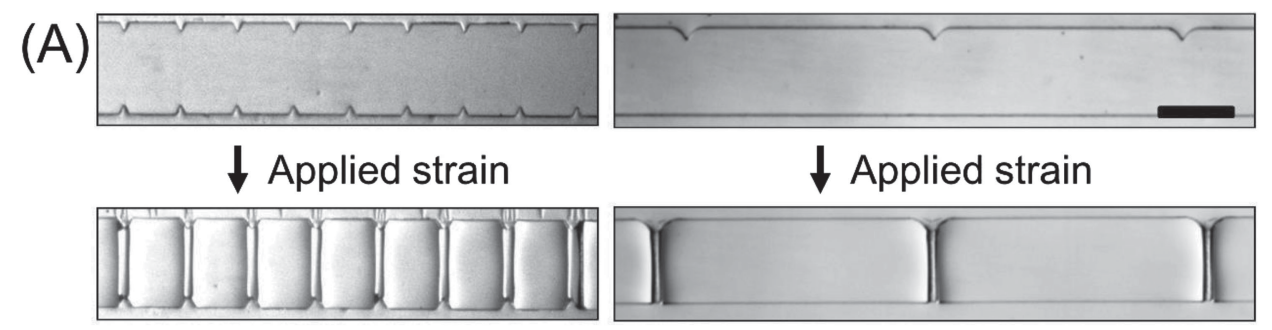

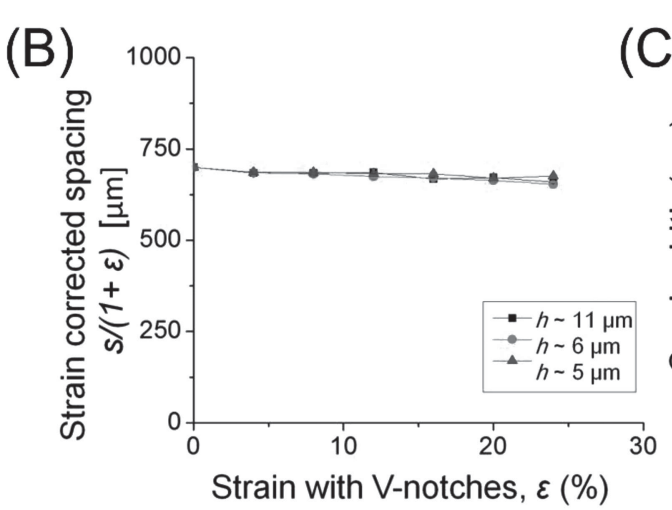

(B)

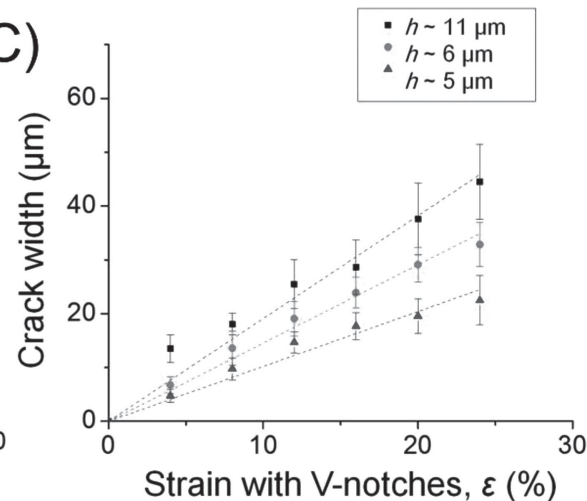

Figure 4. Crack position on the device surfaces can be predictively controlled by incorporating V-notch microstructures into the h-PDMS/PDMS substrates. Cracks are initiated at these points because the notches shield any intrinsic flaws lying between them. ${ }^{[21]} \mathrm{A}$ ) V-notches are fabricated at distinct spacings and an applied strain generates cracks at those locations (scale bar $=200 \mu \mathrm{m}$ ). B) For V-notches spaced $700 \mu \mathrm{m}$ apart, cracks can be formed at the notch sites. Applied widening strains up to $25 \%$ then provide a stable, normalized spacing without generating additional cracks, enabling the formation of adjustable crack structures at specified locations on the substrate. C) The cracks at these precisely defined locations have well-controlled widths that depend on the applied strain (linear fit $R^{2}$ value $>0.97$ for all data sets). Hence, the position and width of the reversible cracks can be prescribed accurately. 
as those needed for many microfluidic devices, indicating that this system may be useful for a number of applications in developing adjustable microfluidic channels and systems.

\subsection{Design of Normally Closed Microfluidic Systems}

To demonstrate the potential applications of this system to the development of adjustable microscale fluidic platforms, we fabricated a simple geometrically controllable and fully reversible microfluidic channel. To achieve this, a pre-cracked brittle h-PDMS/PDMS bilayer was sealed against a PDMS slab (Figure 5A). Applied tension enlarges the cracks in the sandwiched layer, enabling control of crack width by varying the applied strain. A fluorescent solution of Rhodamine B was passed through the crack structures to enable clear visualization of channel dimensions (Figure 5B) and cross-section (Figure 5C). The crack cross-section follows the expected profile shown in Figure 2,3. Vertical asymmetry in the channel profile is due to the fabrication procedure: cracks were generated in the h-PDMS/PDMS bilayer first, before bonding the second PDMS layer to seal the channel, resulting in a flat profile on one side, and a parabolic profile on the other. Additional experiments in which the cracks were generated after forming the tri-layer showed the symmetry in the confocal images expected for two crack tips (data not shown).

Releasing the applied strain drove the dye out of the system. In the case of non-adsorbing dyes, such as food coloring in water (Figure 5D), the liquid was completely removed from the channel and could no longer be detected by image analysis (Figure 5E). Hence, microfluidic channels that are normally closed and completely reversible can be fabricated using this technique.

The ability of the system to completely heal a generated crack is of particular importance in applications that typically require microfluidic valves. Typically, valve structures require complex fabrication procedures to create hemispherical channels ${ }^{[1]}$ or bell-shaped channels ${ }^{[29,30]}$ to allow the deformation of one of the channel walls to form a leak-proof seal. Such valves provide significant advantages such as highly-localized actuation capabilities, but are also challenging to mass produce, requiring careful alignment techniques or workarounds, ${ }^{[31]}$ expensive operating equipment, and specialized expertise in multilayer soft lithography. ${ }^{[5]}$ While the fracturefabricated adjustable microchannels presented in this work cannot provide highly localized and individually addressable valve actuation, they may significantly improve the fabrication process and workflow for applications involving simultaneous operation of multiple valves. To demonstrate the potential of this technology in such applications, we fabricated diamondshaped cavities within the h-PDMS layer (Figure 5F). The cavities simultaneously direct the formation of cracks through stress concentration, and can serve as reaction chambers or compartments. Either rows or columns of chambers can be connected serially via uniaxial applied strains, or simultaneously connected across all rows and columns via biaxial strains. Furthermore, our microscale structures heal completely once the applied strain is removed, without the specialized fabrication considerations and operating equipment required in most systems requiring microfluidic valves. This ability to selectively address and compartmentalize rows or columns of reaction chambers may be applied to various microfluidic applications, such as single-cell trapping for analysis or culture, highthroughput reaction screens, or for analytical applications requiring single-molecule compartmentalization.

The relatively flat-bottomed crack profiles observed (Figure 3,5C) may also be a significant advantage in many microfluidic applications. Cracks in this h-PDMS/PDMS system display a more rectangular shape than the sharp parabolic profiles demonstrated in gold/PDMS systems. ${ }^{[20]}$ This may be of importance for applications involving microscopy, particularly of cultured cells. The presence of a flat culture surface would enable simple microscopy-based image collection and analysis of cells cultured in the adjustable microfluidic structures. In contrast, parabolic crack profiles present potentially undesirable and undefined topographical cues to cultured cells, and necessitate the use of complex and expensive analytical tools and equipment such as confocal or autofocusing microscopes. The flat culture surfaces also simplify calculations and modeling of fluid flow for potential studies involving the application of flow-induced shear stress on cultured cells. ${ }^{[32]}$

Although the characterization data presented in Figure 7, 4 is limited to relatively small microfluidic channels, the working principles of this technique are applicable to generate a wide variety of channel dimensions. Beyond the specifications of the present system, silicones that can support greater ultimate tensile strains may be used to generate wider channels. The h-PDMS may be processed into thicker layers to create deeper channels, and may be blended with other polymers to increase material toughness and reduce any unintentional cracking during sample handling. Furthermore, the methods used in the preparation of these substrates are compatible with recent interest in DIY (do-it-yourself) "garage microfluidics", and the ability to conduct microfluidic experiments with minimal equipment, training and facilities. Replacing oxidized PDMS with h-PDMS as the brittle layer eliminates the need for expensive and specialized plasma oxidation systems, and adjustable microfluidic channels can be fabricated using a spin-coater and a weigh scale to measure PDMS and h-PDMS components. While we chose to spincoat the h-PDMS layer onto the PDMS slabs, this process can easily be replaced by the industrially-used spreading process, in which the h-PDMS is coated on the surface and smoothed with a blade; a procedure which does not require specialized equipment, and can be done by hand. Hence, this procedure is applicable in a variety of contexts, including resource-limited settings. Finally, because the h-PDMS layer is mechanically tougher than oxidized PDMS layers, it is experimentally easier to handle the substrates without causing undesired cracking due to unintentional mechanical deformation, making this technique both reliable and experimentally simple.

\subsection{Application: Mechanical Lysis of Single Cells and Manipulation of Nuclear Chromatin}

As a first demonstration of the unique utility of this technology to biological studies, we used the normally closed 

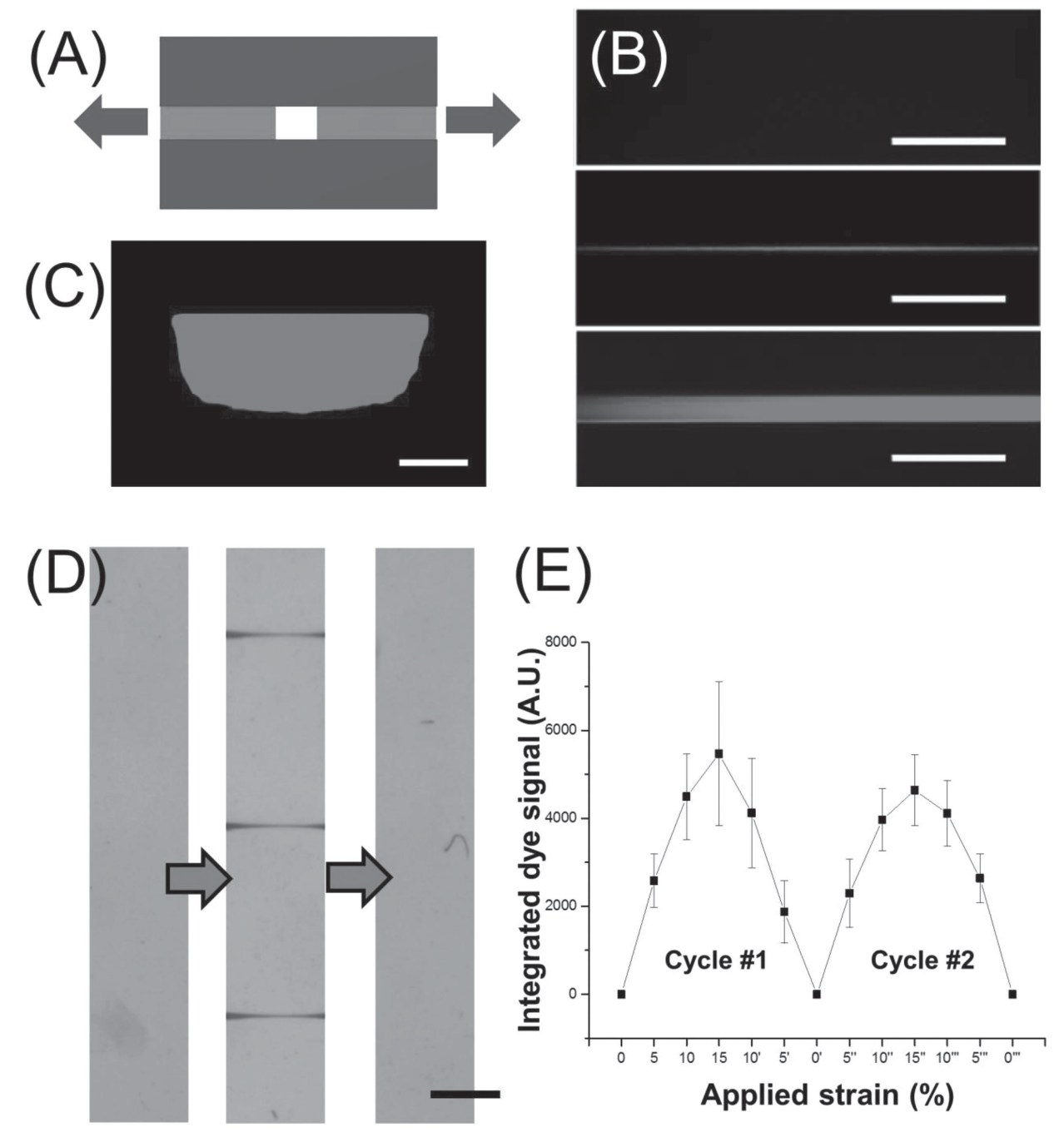

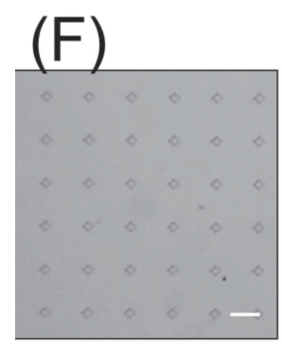

Relaxed
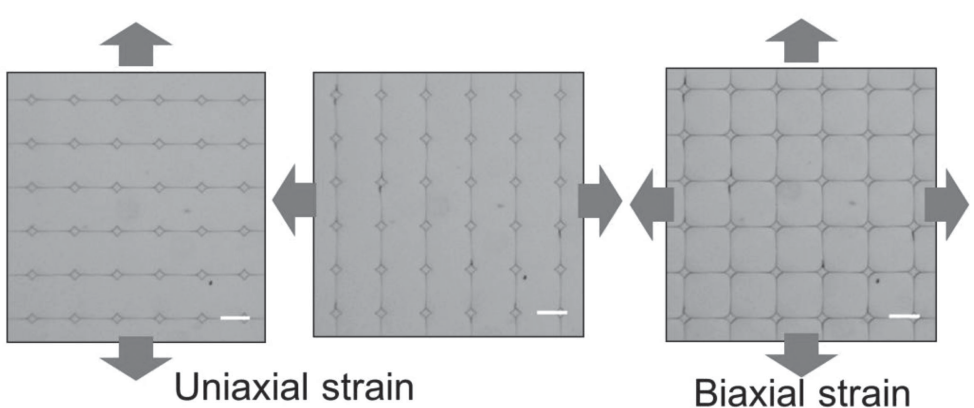

Figure 5. Adjustable and reversible microfluidic structures. A) A schematic cross-section demonstrating the spontaneous formation of a microfluidic crack structure within an h-PDMS layer embedded in a PDMS substrate. B) Fluorescent dye is flowed through the microfluidic structures to demonstrate fluidic connections and the ability to adjust the size of fracture-based channels. Scale bar: $50 \mu \mathrm{m}$. C) Representative confocal image of the cross-sectional area of a fracture-fabricated microfluidic channel at $20 \%$ strain, filled with fluorescent dye. Scale bar: $5 \mu$ m. D) Optical micrograph showing that multiple cracks can be simultaneously generated and reversibly closed to expel liquid from the microfluidic channels. Scale bar: $100 \mu \mathrm{m}$. E) Integrated signal from the red dye is measured over two repeated open-and-close strain cycles, demonstrating that no measurable level of liquid remains within the channel after closure. This finding further establishes that no delamination occurs between the material layers. F) Diamond-shaped microfabricated cavities in the h-PDMS layer may be used to simultaneously direct crack formation and provide addressable fluid compartments for a variety of applications requiring valved reaction chambers.

microscale fluidic channels to mechanically lyse individual cells and manipulate the chromatin released from the nucleus, for subsequent epigenetic analysis of single cells. This process is demonstrated in Figure 6. The PDMS/h-PDMS/ PDMS system enables the generation of channels that can be reversibly opened to widths of 10 s of micrometers, and these channels are large enough to accept a dilute suspension of single cells. Once a single cell is positioned in the channel, the channel is collapsed by slowly reducing the external tension on the device. The collapsing channel walls pin, compress 

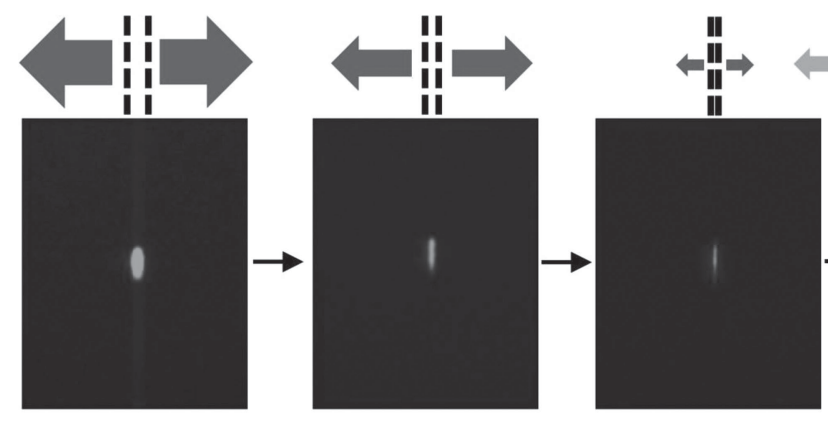

Cell lysis by mechanical compression

Figure 6. Application of adjustable reversible microstructures to lyse single cells and manipulate released nuclear chromatin. A single HeLa cell with a GFP-labeled H2B histone is trapped in enlarged crack-fabricated microchannels and lysed by compression applied via tension release. Once lysed, the channel is opened and closed, forcing the GFP-labeled chromatin to linearize due to elongational shear forces imposed by the fluid, ${ }^{[11]}$ thereby confirming cell lysis. Scale bar $=25 \mu \mathrm{m}$.

and lyse the cells, releasing the chromatin complex from the cell nucleus. The channel is then opened and rapidly closed, generating elongational squeezing flows that linearize the released chromatin ${ }^{[11]}$ (Figure 6). As discussed in our previous work, ${ }^{[11]}$ elongational flows along one dimension of a channel as well as simultaneous constraints presented along the other two dimensions are necessary to linearize chromatin. These dynamic mechanical constraints cannot be replicated in conventionally deformable mechanical lysis chambers, which are hence unsuitable for the structural manipulation of chromatin. ${ }^{[33]}$ For visualization purposes, the cells used in this demonstration have been transfected to stably express fluorescently labeled histones (H2B) in the chromatin complex. While the degree of chromatin linearization relative to its contour length is small in this initial demonstration, it is noted that we have previously demonstrated the ability to linearize chromatin strands to a greater degree using nanoscale fracture-based channels. ${ }^{[11]}$ However, this previous nanoscale chromatin linearization study ${ }^{[11]}$ required chromatin materials from many cells to be pooled together to allow for liquid handling needs. The ability to construct both microscale and nanoscale channels by fracture opens the way for future studies with devices that integrate microscale singlecell lysis followed by nanoscale chromatin linearization and mapping, to study the epigenetic structure of chromatin at the single-cell level. Such approaches are particularly valuable in studying stem cells and other rare cell populations, and may provide significant advantages over conventional chromatin immunoprecipitation (ChIP) assays that require many cells and evaluates one histone modification at a time. ${ }^{[14]}$

\section{Conclusions}

The use of hard PDMS (h-PDMS) as a brittle layer in multilayered structures for crack fabrication provides distinct advantages over more conventionally used oxidized PDMS or metal systems. While the use of cracking in multilayered materials under tension has previously been proposed as a

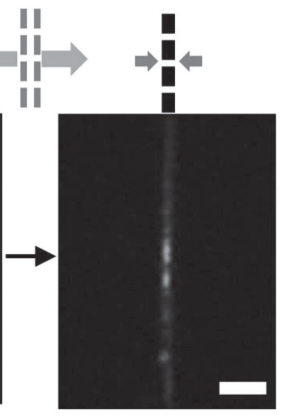

Chromatin manipulation novel nano-fabrication paradigm, significant challenges exist in defining microscale dimensions of cracks in bilayer material systems. h-PDMS can be processed into a layer of defined thickness at the micrometer length scale by spin-coating, and produces an array of stable flat-bottomed cracks under tension when supported on an underlying tough PDMS substrate. The toughness mismatch between h-PDMS and PDMS is large enough to generate stable crack arrays at relatively low strains, yet the modulus mismatch is small enough to strongly localize the cracks to the h-PDMS layer. Hence, the crack depth can be controlled based on the thickness of the h-PDMS layer. Microfabricated crack-initiating structures can be introduced into the material, enabling the precise positioning of cracks in this system. The crack width can also be tuned by varying the applied strain. In this way, precisely defined crack dimensions can be prescribed and realized. We have successfully demonstrated the use of this technology to create adjustable, completely-reversible and normally closed microfluidic channels; a particularly challenging structure to fabricate using conventional approaches. We then demonstrated a simple biological application of the system by using the microscale adjustable channels to mechanically lyse single cells, and release and manipulate chromatin. This application requires micrometer-scale channels capable of accepting single cells, and the ability to completely close the channels to ensure mechanical lysis; features that cannot be robustly achieved using existing material bilayer systems. The technologies presented in this work are simple, scalable, inexpensive and require relatively little microfabrication experience, enhancing the potential for microfabricated systems to be used by non-specialists in the microfluidics and precision-fabrication fields.

\section{Experimental Section}

Multilayer Sample Preparation: PDMS elastomers were prepared and cured on glass slides whose surface had been rendered non-adhesive to PDMS by exposure to the vapor phase of the silanization agent (tridecafluoro-1,1,2,2-tetra-hydrooctyl)-1-trichlorosilane (United Chemical Technologies) for 30 min (Figure 7a). h-PDMS was prepared following established protocols. ${ }^{[34]} 3.4 \mathrm{~g}$ of vinyl PDMS pre-polymer (VDT-731; Gelest Corp.), $18 \mu \mathrm{L}$ of a platinum catalyst (platinum-divinyltetramethyldisiloxane, SIP6831.2, Gelest Corp.) and a drop of a modulator (2,4,6,8-tetramethyltetravinylcyclotetrasiloxane, 396281, Sigma-Aldrich) were mixed and degassed for several minutes. To initiate polymerization, one gram of a hydrosilane prepolymer (HMS-301; Gelest Corp.) was added; the system was mixed thoroughly and degassed for one minute. The h-PDMS was then diluted in hexane $(20 \%$ or $50 \%$ $\mathrm{w} / \mathrm{w}$ depending on the desired thickness) to reduce the viscosity of the fluid, and spin-coated onto the silanized glass slide, using different spin times and speeds to control the thickness of the 

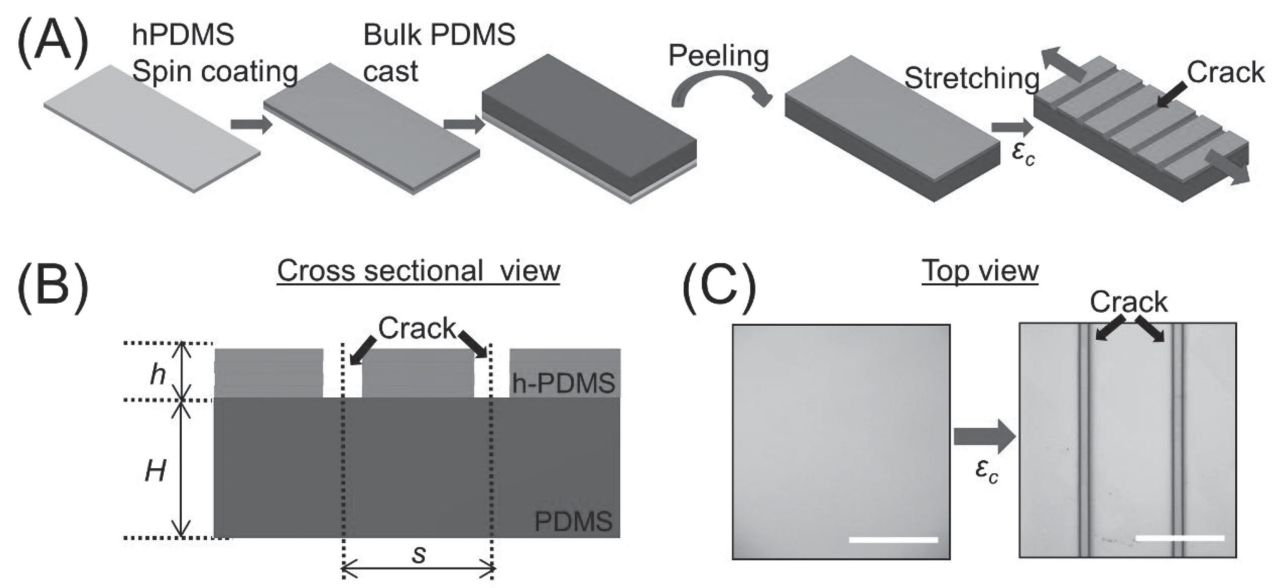

Figure 7. Experimental schematic. A) The fabrication process. A thin h-PDMS layer is spin coated onto a silanized glass slide and partially cured. PDMS is then cast on top of the film and fully cured. The bilayer is peeled away from the glass slide, and stretched to generate an array of cracks. B) The dimensioning nomenclature. The thickness of the h-PDMS layer and bulk PDMS are denoted by $h$ and $H$ respectively, and the crack spacing $s$ is measured from center to center of the troughs. C) A representative bright-field image of crack formation in the substrate under an applied strain. Scale bar $=50 \mu \mathrm{m}$.

h-PDMS layer. The relationship between film thickness, spin-time and speed was characterized using a laser interferometer (LEXT, Olympus OLS4000). Combinations of three different spin speeds (2000 rpm, $4000 \mathrm{rpm}$, and $6000 \mathrm{rpm}$ ) and four different spin times $(30 \mathrm{~s}, 60 \mathrm{~s}, 120 \mathrm{~s}$, and $600 \mathrm{~s})$ were employed to generate films of various thicknesses. This layer was then partially cured in an oven at $120^{\circ} \mathrm{C}$ for one minute to stabilize the liquid film.

Standard PDMS elastomer was prepared using a Sylgard 184 kit (Dow Corning) by mixing the monomer and cross-linking components in a 10:1 ratio. The resulting mixture was degassed, and cast to a thickness of $5 \mathrm{~mm}$ over the partially cured h-PDMS layer. The entire system was then cured at $60{ }^{\circ} \mathrm{C}$ overnight, before the h-PDMS/PDMS bilayer was peeled from the glass slide, and stored at room temperature until use.

Materials Characterization: The Young's moduli of the PDMS and h-PDMS were measured using uniaxial tensile tests and compression tests. The tensile specimens were prepared by casting PDMS and h-PDMS in dog-bone shaped molds, following ASTM E1820-11e2. After release from the molds, the specimens were clamped in wedge grips. The tensile tests were performed at room temperature using an MTS 858 Bionix II tensile machine. The load was applied at a constant strain rate of $0.0080 / \mathrm{s}$, and the load was measured using a $250 \mathrm{~N}$ load cell. The strains were determined using MetaMorph software to analyze the displacements of markers on the sample that had been recorded optically. True stress-strain curves were used to calculate the Young's modulus of each material. The compressive tests were conducted using a TA XT-PLUS Texture analyzer, set up with a $30 \mathrm{~kg}$ load cell. PDMS and h-PDMS samples were cast and cured to a thickness of 5-10 mm in $22 \mathrm{~mm}$ diameter containers. A stainless steel spherical indenter of radius $6.35 \mathrm{~mm}$ was used to apply a deformation of $250 \mu \mathrm{m}$ into the material. The resulting force-displacement curves were fitted to a Hertzian spherical indentation model to calculate the modulus of the material. For the compression tests, three indentation curves were generated from each of at least three independent samples, and averaged. The toughness of the h-PDMS was measured using a tensile specimen with an edge crack that had been introduced by a razor blade. The crack was imaged optically during the tensile test (Supporting Information Movie 1). No sub-critical crack growth was observed, so the peak load and the original crack length were used to determine the toughness. The toughness of the standard PDMS was previously reported by Mills et al. using a compact-tension specimen. ${ }^{[17]}$

Crack Generation and Analysis: After peeling from the glass slides, the multilayer samples were loaded into a MicroVice Holder (S.T. Japan USA LLC. FL, USA). The MicroVice is a microscope-compatible device capable of manually applying uniaxial strains to the sample. These applied strain generated the cracks used as microchannels. Where orthogonal arrays of channels were required, biaxial strains were applied using a homemade biaxial stretching system, built by assembling the component parts of two MicroVice sample holders on a custom-fabricated acrylic plate. The applied strains were measured using digital calipers with a resolution of $0.01 \mathrm{~mm}$, and the resulting crack dimensions were determined using a laser interferometer (LEXT, Olympus OLS4000). Recorded characteristics included the crack width and depth, and the average spacing between cracks.

Modeling Crack Deformation: The cross-sectional profiles of cracks in the h-PDMS/PDMS system were simulated using a hyperelastic material model in the commercially available finite-element analysis software ABAQUS (Dassault Systèmes). The model simulated a $7.2 \mu \mathrm{m}$ thick layer of h-PDMS bonded to a $5 \mathrm{~mm}$ thick layer of PDMS, under applied strains of 25 and 35\%. The depth of crack penetration into the PDMS layer was iteratively determined by fitting to the experimental data for the crack profile.

Fabrication of Microfeatures within Sealed Bilayer Structures: V-notch shaped stress concentrators, ${ }^{[21,35]}$ arrayed diamondshaped chambers, and micropatterned features for fluid flow were fabricated into the h-PDMS layer by spin-coating the h-PDMS layer onto a microfabricated SU-8 (Microchem) master structure fabricated on a silicon wafer using standard photolithography. After the h-PDMS layer was partially cured, a layer of standard PDMS was cast over the mold and cured overnight at $60^{\circ} \mathrm{C}$. The h-PDMS/PDMS bilayer was then carefully peeled from the mold, and stretched to generate cracks. The h-PDMS side of the bilayer was then plasma oxidized and placed in conformal contact with a 
similarly treated slab of cured PDMS and allowed to bond covalently, forming the PDMS/h-PDMS/PDMS sandwich structure. To characterize the sealed channels, Rhodamine B solution was loaded into the microchannels so that the crack dimensions could be measured directly by confocal fluorescent microscopy. Alternatively, to avoid binding of hydrophobic dyes to the PDMS channel walls, a food-coloring dye was diluted in a mixture of water and ethanol, loaded into the channels, and imaged under varying degrees of strain.

Mechanical Lysis of Cells: HeLa cells were cultured in growth medium (Dulbecco's Modified Eagle Medium (DMEM) supplemented with $10 \%$ Fetal Bovine Serum and 1\% antibiotics-antimycotics), and stably transfected to express a green-fluorescent $\mathrm{H} 2 \mathrm{~B}$ histone protein as previously described. ${ }^{[11]}$ To conduct the lysis experiments, cells were trypsinized, centrifuged and resuspended at a density of $\sim 10^{5}$ cells $/ \mathrm{mL}$. Cells were loaded into parallel microchannels fabricated into the multilayered h-PDMS/PDMS material, and tension was applied in the same direction as the microchannels. Cracks connecting the microfluidic channels were generated, and single cells were driven into the cracked channels by slow flow. Once in the channels, the tensile strain was slowly released to pin and mechanically lyse the cell within the microfluidic channel. Cell lysis was confirmed by rapidly opening and closing the channel to generate elongational fluid flows ${ }^{[11]}$ and linearize the released chromatin. Linearized chromatin was visualized using standard fluorescent microscopy with a 40x objective.

\section{Supporting Information}

Supporting Information is available from the Wiley Online Library or from the author.

\section{Acknowledgements}

B.C.K. and C.M. contributed equally to this work. B.C.K., C.M., and T.M. fabricated the h-PDMS/PDMS devices and collected and analyzed data. C.M. and J.H. conducted the mechanical characterizations and analyses. C.M. conducted cell culture studies. M.D.T. guided the understanding of the mechanics issues, and helped provided guidance for the direction and design of the project. S.T. was responsible for the overall design and direction of the project. All authors contributed to the design and interpretation of the experiments, and edited the manuscript. The authors gratefully acknowledge support from the Natural Sciences and Engineering Research Council of Canada, and from the Banting postdoctoral fellowship programs to C.M. This work was supported by a grant from the US National Institutes of Health (HG004653-03) and a Rare Cells Seed Grant from the Biointerfaces Institute, University of Michigan.

[1] M. A. Unger, H.-P. Chou, T. Thorsen, A. Scherer, S. R. Quake, Science 2000, 288, 113-116.

[2] W. H. Grover, A. M. Skelley, C. N. Liu, E. T. Lagally, R. A. Mathies, Sens. Actuators B Chem. 2003, 89, 315-323.
[3] A. R. Abate, M. B. Romanowsky, J. J. Agresti, D. A. Weitz, Appl. Phys. Lett. 2009, 94, 023503.

[4] W. Gu, X. Zhu, N. Futai, B. S. Cho, S. Takayama, Proc. Natl. Acad. Sci. U.S.A. 2004, 101, 15861-15866.

[5] J. Melin, S. R. Quake, Annu. Rev. Biophys. Biomol. Struct. 2007, $36,213-231$.

[6] Y. Sun, L.-T. Jiang, R. Okada, J. Fu, Langmuir 2012, 28, 10789-10796.

[7] A. Lenshof, T. Laurell, Chem. Soc. Rev. 2010, 39, 1203-1217.

[8] X. Zhu, K. L. Mills, P. R. Peters, J. H. Bahng, E. H. Liu, J. Shim, K. Naruse, M. E. Csete, M. D. Thouless, S. Takayama, Nat. Mater. 2005, 4, 403-406.

[9] D. Huh, K. L. Mills, X. Zhu, M. A. Burns, M. D. Thouless, S. Takayama, Nat. Mater. 2007, 6, 424-428.

[10] T. Uchida, K. L. Mills, C.-H. Kuo, W. Roh, Y.-C. Tung, A. L. Garner, K. Koide, M. D. Thouless, S. Takayama, Langmuir 2009, 25, 3102-3107.

[11] T. Matsuoka, B. C. Kim, J. Huang, N. J. Douville, M. D. Thouless, S. Takayama, Nano Lett. 2012, 12, 6480-6484.

[12] A. R. Dixon, C. Moraes, M. E. Csete, M. D. Thouless, M. A. Philbert, S. Takayama, J. Biomed. Mater. Res. A 2013, DOI: 10.1002/ jbm.a.34814.

[13] R. Chantiwas, S. Park, S. A. Soper, B. C. Kim, S. Takayama, V. Sunkara, H. Hwang, Y.-K. Cho, Chem. Soc. Rev. 2011, 40, 3677.

[14] T. Matsuoka, B. Choul Kim, C. Moraes, M. Han, S. Takayama, Biomicrofluidics 2013, 7, 041301.

[15] B. C. Kim, C. Moraes, J. Huang, M. D. Thouless, S. Takayama, Biomater. Sci. 2014, 2, 288.

[16] C. Moraes, B. C. Kim, X. Zhu, K. L. Mills, A. Dixon, M. D. Thouless, S. Takayama, Lab Chip 2014, DOI: 10.1039/C4LC00122B.

[17] K. L. Mills, X. Zhu, S. Takayama, M. D. Thouless, J. Mater. Res. 2008, 23, 37-48.

[18] S. Béfahy, P. Lipnik, T. Pardoen, C. Nascimento, B. Patris, P. Bertrand, S. Yunus, Langmuir 2010, 26, 3372-3375.

[19] W. W. Tooley, S. Feghhi, S. J. Han, J. Wang, N. J. Sniadecki, J. Micromech. Microeng. 2011, 21, 054013.

[20] N. J. Douville, Z. Li, S. Takayama, M. D. Thouless, Soft Matter 2011, 7, 6493.

[21] B. C. Kim, T. Matsuoka, C. Moraes, J. Huang, M. D. Thouless, S. Takayama, Sci. Rep. 2013, 3, 3027.

[22] M.-C. Cheng, A. T. Leske, T. Matsuoka, B. C. Kim, J. Lee, M. A. Burns, S. Takayama, J. S. Biteen, J. Phys. Chem. B 2013, 117, 4406-4411.

[23] S. P. Lacour, S. Wagner, Z. Huang, Z. Suo, Appl. Phys. Lett. 2003, 82, 2404.

[24] T. Adrega, S. P. Lacour, J. Micromech. Microeng. 2010, 20, 055025.

[25] M. D. Thouless, Z. Li, N. J. Douville, S. Takayama, J. Mech. Phys. Solids 2011, 59, 1927-1937.

[26] H. Schmid, B. Michel, Macromolecules 2000, 33, 3042-3049.

[27] E. Kang, J. Ryoo, G. S. Jeong, Y. Y. Choi, S. M. Jeong, J. Ju, S. Chung, S. Takayama, S.-H. Lee, Adv. Mater. 2013, 25, 2167-2173.

[28] N. Futai, W. Gu, S. Takayama, Adv. Mater. 2004, 16, 1320-1323.

[29] D. Lai, J. M. Labuz, J. Kim, G. D. Luker, A. Shikanov, S. Takayama, RSC Adv. 2013, 3, 19467-19473.

[30] C. Moraes, Y. Sun, C. A. Simmons, J. Micromech. Microeng. 2009, 19, 065015.

[31] C. Moraes, Y. Sun, C. A. Simmons, Integr. Biol. 2011, 3, 959-971.

[32] Y. C. Kim, J. H. Kang, S.-J. Park, E.-S. Yoon, J.-K. Park, Sens. Actuators B Chem. 2007, 128, 108-116.

[33] J. Huang, B. C. Kim, S. Takayama, M. D. Thouless, J. Mater. Sci. 2014, 49, 255-268.

[34] T. W. Odom, J. C. Love, D. B. Wolfe, K. E. Paul, G. M. Whitesides, Langmuir 2002, 18, 5314-5320.

[35] J. L. Beuth, N. W. Klingbeil, J. Mech. Phys. Solids 1996, 44, 1411-1428.

Received: January 17, 2014

Revised: April 30, 2014

Published online: June 18, 2014 